\title{
Un nuevo caso de "schwannoma del olfatorio"; presentación y revisión de la literatura
}

\author{
L. Martínez-Soto; R. Alfaro-Baca; M.V. Torrecilla-Sardón; B. Fernández-Vallejo; R. Ferreira-Muñóz y T. De Diego*
}

Servicio de Neurocirugía y Anatomía Patológica*. Hospital Santiago Apóstol. Vitoria. España.

\section{Resumen}

Presentamos el caso de un paciente varón de 54 años que acude a Urgencias por cefalea importante de 6 días de evolución así como por una disfasia nominal ocasional. No presentaba anosmia ni otra sintomatología. El TAC urgente y la RM craneal posterior muestran una gran tumoración extra-axial frontobasal de $7 \times 6 \times 5 \mathrm{~cm}$ de diámetro, localizada mas sobre el lado derecho de la lámina cribosa, que capta contraste hetereogéneamente y cuyo diagnóstico de sospecha fue meningioma del surco del olfatorio. Tras su exéresis total mediante craneotomía bifrontal, la anatomía patológica es informada como schwanoma de tipo convencional. Dada la rareza de este tipo de tumores (26 hasta la fecha), revisamos la literatura, sus posibles diferencias radiológicas con el meningioma del surco del olfatorio y las diversas teorías acerca de su origen.

PALABRAS CLAVE: Schwanoma. Surco del olfatorio. Nervio olfatorio.

A new case of "olfactory schwannoma"; presentation and literature review

\section{Summary}

We report the case of a 54-year-old man who presented at the Emergency Department with intense headache of 6-days duration and sporadic nominal dysphasia. He did not present anosmia and the rest of the examination was normal. The emergency $C T$ and the posterior cerebral MR showed a great subfrontal extra-axial mass of $7 \times 6 \times 5 \mathrm{~cm}$, over the right side of the cribiform plate, hetereogeneously enhancing after gadolinium administration. Preoperative diagnosis was olfactory groove menigioma. After total removal by bifrontal craniotomy the histopathological diagnosis was schwannoma of the conventional type. Owing to

Recibido: 20-05-08. Aceptado: 30-06-08 the unusual frequency of this kind of tumors (26 to the date), we review the literature, the possible radiological differences with olfactory groove meningiomas and the different theories about their origin.

KEY WORDS: Schwannoma. Olfactory groove. Olfactory nerve.

\section{Introducción}

Los schwannomas son tumores benignos originados a partir de las células de Schwann de los nervios periféricos y de la porción distal de los nervios craneales. En el cráneo aparecen normalmente de la porción vestibular del VIII par y, más raramente, del V, IX y III pares. El nervio olfatorio no tiene células de Schwann y por lo tanto no desarrolla schwannomas.

La rareza de estos tumores (26 casos encontrados en la literatura hasta la fecha) y el dudoso origen de los mismos nos ha llevado a presentar este nuevo caso y, revisando la literatura existente respecto a los casos previos, intentar establecer alguna diferencia radiológica que nos pueda ayudar en el diagnóstico preoperatorio y entender la causa de su aparición en esta localización tan peculiar.

\section{Caso clínico}

Varón de 54 años que acude al Servicio de Urgencias por presentar 6 días antes un episodio de cefalea brusca, de intensidad superior a la habitual, que persiste hasta el momento de la consulta. Además refiere varios episodios de dificultad para denominar objetos. El resto de la exploración neurológica era normal, destacando la ausencia de anosmia.

Se realiza un TAC craneal urgente donde se aprecia una gran masa en región froto-basal de predominio derecho de $7 \times 6 \times 5 \mathrm{~cm}$, extra-axial con centro hipodenso, que capta contraste de manera importante, sobre todo en la periferia; no se apreciaban alteraciones del hueso de la fosa craneal anterior (Fig. 1).

La RM posterior muestra la masa extra-axial de aspecto 

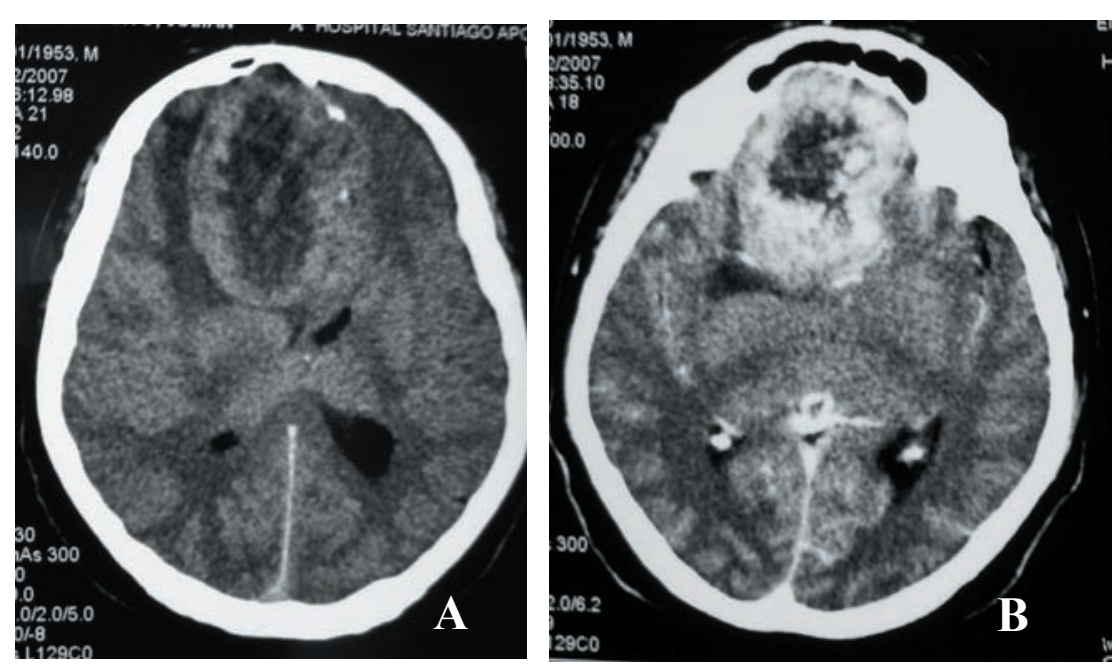

2009 20: 294-297

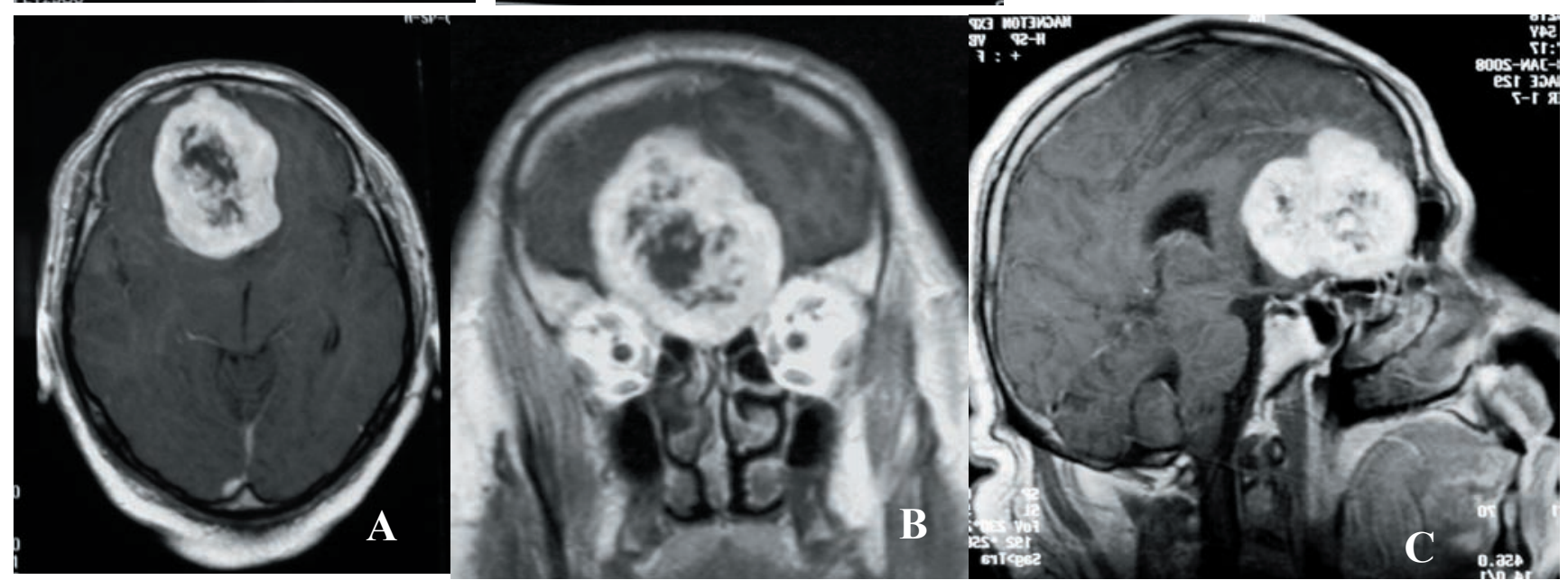

Figura 2. La RM craneal axial A), coronal B) y sagital C) con contraste intravenoso, muestra el tumor subfrontal localizado en el surco del olfatorio en relación con la lámina cribosa del etmoides y con captación de contraste heterogénea, sobre todo en la periferia.
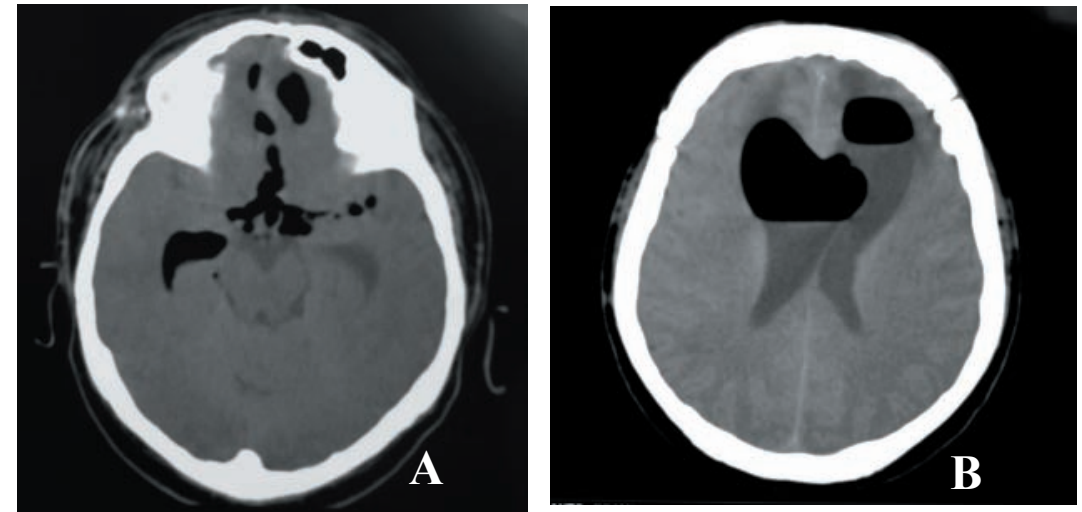

Figura 1. En el TAC craneal de Urgencias $\sin$ A) y con contraste B) se aprecia una tumoración subfrontal, aparentemente extra-axial y que capta contraste de manera heterogénea. heterogéneo, bordes bien definidos, con áreas de hiperseñal en T2 que sugieren necrosis/degeneración quística, así como otros focos hipointensos en $\mathrm{T} 2$ compatibles con focos hemorrágicos. Capta contraste de manera heterogénea de predominio periférico. Está en contacto con la hoz cerebral y con el surco del olfatorio. La imagen es informada como
Figura 3. El TAC craneal un mes después de la cirugía muestra aire en las cisternas de la base A) y en el sistema ventricular B). 
óseo de 3-4 mm en su estructura. No se encontraron durante la cirugía los nervios olfatorios. Se procede a tapizar la lámina cribosa con el periostio frontal que se refleja y con él cubrimos también el seno frontal cranealizado. Como única complicación presenta una fístula de LCR de escasa importancia; es dada de alta a los siete días. El paciente reingresa al mes por cuadro de hipertensión intracraneal y reaparición de la fístula de LCR y en el TAC se aprecia aire en cisternas de la base y en ventrículos (Fig. 3); el cuadro se soluciona con un drenaje ventricular y posteriormente una derivación lumboperitoneal.

La anatomía patológica es informada como un tumor con una población celular de hábito fusiforme y con empalizadas nucleares. La inmunohistoquímica reveló positividad para Vimentina, Proteína S-100 y negativa para EMA. Todo esto nos llevo al diagnóstico de Schwanoma.

\section{Discusión}

Los schwannomas intracraneales no asociados con los pares craneales son menos del 1\% de los schwannomas del sistema nervioso central y periférico tratados quirúrgicamente. Los schwannomas de la región del olfatorio son tumores extremadamente raros y en la revisión de la literatura realizada solo hemos encontrado 26 casos descritos no relacionados con la Enfermedad de von Recklinghausen $^{1,2,5,8}$; el primer caso citado en la literatura inglesa lo publicaron Gibson et al en 1966.

Por definición, los schwannomas se originan de las células de Schwann y el nervio olfatorio, como parte del sistema nervioso central, no contiene células de Schwann. Por lo tanto, los " schwannomas del olfatorio" no serían realmente tumores derivados del nervio olfatorio y su relación física con dicho nervio no esta demostrada ${ }^{4}$.

Como se aprecia en la revisión de Adachi K. et $\mathrm{al}^{1}$, la edad media de presentación de estos tumores es menor $(32.2 \pm 11.9)$ que el caso presentado y en general menor que el resto de schwannomas intracraneales. Además, la mayoría de los pacientes no referían anosmia en su presentación clínica, lo cual confirma su falta de relación con el nervio olfatorio.

Otra de las peculiaridades de este tipo de tumores es que su diagnóstico preoperatorio en la casi totalidad de los casos fue de meningioma del surco del olfatorio. Esto suele ser debido, en primer lugar, a su rareza y falta de sospecha como primer diagnóstico $\mathrm{y}$, en segundo lugar, a que sus características radiológicas no permitían distinguirlo de un meningioma de dicha zona. Pero en los distintos casos publicados podemos encontrar algunos datos radiológicos comunes que nos podrían hacer sospechar la presencia de un neurinoma en vez de un meningioma. Uno de estos datos es que, en la mayoría de los casos, el tumor aparecía como quístico en el TAC y en la RM y con captación heterogénea del contraste. Otro de los rasgos es el hecho de que, sobre todo en el TAC, se aprecie una alteración a nivel de la lámina cribosa del etmoides, consistente en erosión ósea con festoneado (scalloped) y destrucción ósea ${ }^{10}$; en los meningiomas, por el contrario, la afectación ósea suele ser de tipo esclerosis e hipertrofia ${ }^{6}$. En las imágenes de la RM la distinción suele ser más difícil ya que la intensidad de señal tanto en las secuencias T1 como en T2 suele ser similar; quizás, la ausencia de la cola dural propia de los meningiomas, la menor área de implantación en la duramadre y el ángulo agudo entre el tumor y la dura (los meningiomas suelen tener un ángulo obtuso) nos podrían llevar a sospechar la presencia de un neurinoma en vez de un meningioma ${ }^{12}$.

Como hemos mencionado anteriormente, es difícil explicar la presencia de un tumor derivado de las células de Schwann en una región donde no hay este tipo de células. Dos son las teorías que han intentado explicar la aparición de estos tumores en esta región: las relacionadas con alteraciones del desarrollo y las no relacionadas con alteraciones del desarrollo. Las primeras sugieren una transformación de células mesenquimales piales en células ectodérmicas de $\mathrm{Schwann}^{3}$ o la migración de células de la cresta neural dentro de la sustancia del sistema nervioso central ${ }^{7,9}$, que serían el origen de estos tumores. Por otro lado, los que defienden las teorías no relacionadas con alteraciones del desarrollo, proponen que los neurinomas del olfatorio provienen de células de Schwann presentes normalmente en estructuras adyacentes como son el plexo nervioso perivascular, las ramas meníngeas del trigémino y del nervio etmoidal anterior que inervan la fosa craneal anterior ${ }^{4,7,11}$ o de las fila olfatoria que es sabido que adquieren células de Schwann que se extienden aproximadamente $0.5 \mathrm{~mm}$ mas allá del bulbo olfatorio ${ }^{1}$. A nivel práctico, como no se ha demostrado ninguna de estas dos teorías, se ha usado el sitio de anclaje a la duramadre como el sitio de origen de estos tumores y se han clasificado los neurinomas subfrontales en neurinomas del surco olfatorio y neurinomas subfrontales fuera del surco del olfatorio ${ }^{1}$.

En el caso presentado parece que el tumor se originaba de la duramadre basal en el sitio del surco del olfatorio, sugiriendo que el schwanoma podría haberse originado de los pequeños nervios de la duramadre basal, aunque no se podría descartar su relación con las fila olfatoria ya que su localización anatómica coincide con estas estructuras. Si bien la primera localización explicaría mejor la ausencia de anosmia, los tumores originados de las fila olfatoria podrían no ocasionar anosmia ya que a pesar de que el tumor comprime y desplaza el nervio olfatorio y por eso no lo vemos en la cirugía, la presencia de parte de las fila olfatoria intactas preservarían el olfato en estos pacientes ${ }^{1}$. 


\section{Conclusión}

Los schwannomas del olfatorio son tumores extremadamente raros y, por lo tanto, difíciles de llegar a su sospecha en el diagnóstico preoperatorio; pero hay ciertos hallazgos radiológicos que nos pueden ayudar a incluirlos en el diagnóstico diferencial de lo tumores extra-axiales de localización subfrontal. El origen concreto y la etiología de estos tumores está todavía por determinar.

\section{Bibliografía}

1. Adachi, K., Yoshida, K., Miwa, T., Ikeda, E. Kawase, T.: Olfactory schwannoma; Acta Neurochir (Wien) 2007; 149: 605-610.

2. Ahmad, F.U., Gupta, A., Sharma, M.C., Shukla, G., Metha, V.S.: The enigmatic origin of subfrontal schwannomas: report of a case without hyposmia. Acta Neurochir (Wien) 2006; 148: 671-672.

3. Huang, PP., Zagzag, D., Benjamin, V.: Intracranial schwannoma presenting as a subfrontal tumor: case report. Neurosurgery 1997; 40: 194-197.

4. Praharaj, S.S., Vajramani, G.H., Santosh, V., Dhankar, S.K., Kolluri, S.: Solitary olfactory groove schwannoma: case report with review of the literature. Clin Neurol Neurosurg 1999; 101: 26-28.

5. Prasad, D., Jalali, R., Shet, T.: Intracranial subfrontal schwannoma treated with surgery and $3 \mathrm{D}$ conformal radiotherapy. Neurol India 2004; 52: 248-250.
6. Amador, A.R., Santonja, C., Del Pozo, J.M. Ortiz, L.: Olfactory schwannoma. Eur Radiol 2002; 12: 742-744.

7. Russell, D.S., Rubinstein, L.J.: Pathology of tumors of the nervous system. $5^{\text {th }}(\mathrm{Ed})$. London; Arnold, 1989; pp: 776-777.

8. Sano, H., Hayashi, Y., Hasegawa, M. Yamashita, J.: Subfrontal schwannoma without hyposmia-case report. Neurol Med Chir (Tokyo) 2004; 44: 591-594.

9. Sabel, L.H.W., Trepen, J.L.J.M.: The enigmatic origin of olfactory schwannoma. Case report. Clin Neurol Neurosurg 1995; 97: 187-191.

10. Shenoy, S.N. Raja, A.: Cystic olfactory groove schwannoma. Neurol India 2004; 52: 261-262.

11. Timothy, J., Chakrabarty, A., Rice, A., Marks, P.: Olfactory Groove Schwannoma Revisited. Acta Neurichir (Wien) 1991; 141: 671-672.

12. Yuen, A., Trost, N., McKelvie, P., Webster, J. Murphy, M.: Subfrontal schwannoma: a case report and literature review. Journal of Clinical Neuroscience 2004; 11: 663-665.

Martínez-Soto L.; Alfaro-Baca, R.; Torrecilla-Sardón, M.V.; Fernández-Vallejo, B.; Ferreira-Muñóz, R.; De Diego, T.: Un nuevo caso de "schwannoma del olfatorio"; presentacion y revision de la literatura. Neurocirugía 2009; 20: 294-297.

Correspondencia postal: Dr. Luís Martínez Soto. Hospital Santiago Apóstol. Servicio de Neurocirugía. C/ Olaguibel, 29. 01004 Vitoria-Gasteiz. España. 